\title{
Dustiness and Deagglomeration Testing: Interlaboratory Comparison of Systems for Nanoparticle Powders
}

Yaobo Ding, Burkhard Stahlmecke, Araceli Sánchez Jiménez, Ilse L. Tuinman, Heinz Kaminski, Thomas A. J. Kuhlbusch, Martie van Tongeren \& Michael Riediker

To cite this article: Yaobo Ding, Burkhard Stahlmecke, Araceli Sánchez Jiménez, Ilse L. Tuinman, Heinz Kaminski, Thomas A. J. Kuhlbusch, Martie van Tongeren \& Michael Riediker (2015) Dustiness and Deagglomeration Testing: Interlaboratory Comparison of Systems for Nanoparticle Powders, Aerosol Science and Technology, 49:12, 1222-1231, DOI: 10.1080/02786826.2015.1114999

To link to this article: http://dx.doi.org/10.1080/02786826.2015.1114999
(c) 2015 The Author(s). Published with license by American Fisheries Society (C) Yaobo Ding, Burkhard Stahlmecke, Araceli Sanchez Jimenez, IIse L. Tuinman, Heinz

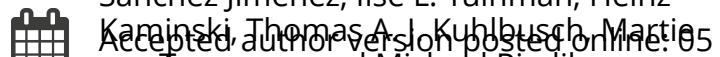 NoD tongeren, and Michael Riediker

Шll Article views: 210

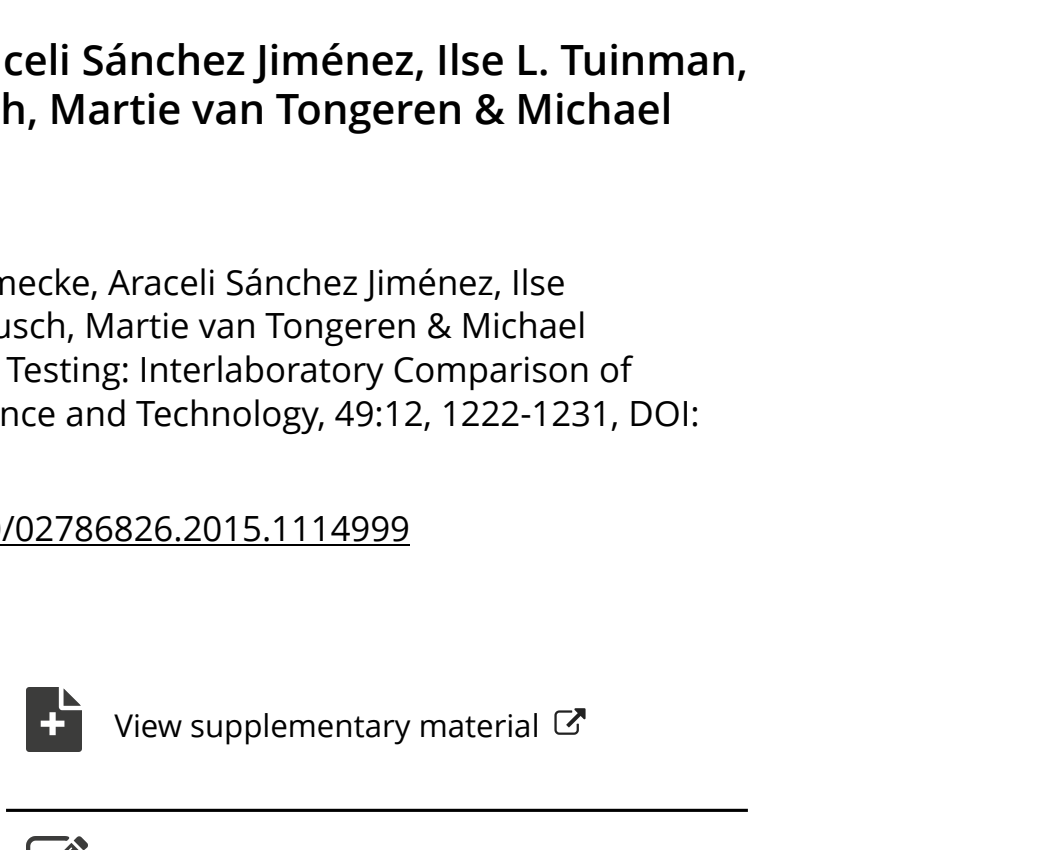

\section{Cons}

View Crossmark data $₫$

Submit your article to this journal $\longleftarrow$

View related articles 


\title{
Dustiness and Deagglomeration Testing: Interlaboratory Comparison of Systems for Nanoparticle Powders
}

\author{
Yaobo Ding, ${ }^{1}$ Burkhard Stahlmecke, ${ }^{2}$ Araceli Sánchez Jiménez, ${ }^{3}$ Ilse L. Tuinman, ${ }^{4}$ Heinz Kaminski, ${ }^{2}$ \\ Thomas A. J. Kuhlbusch, ${ }^{2}$ Martie van Tongeren, ${ }^{3}$ and Michael Riediker ${ }^{1,5}$ \\ ${ }^{1}$ Institute for Work and Health, Universities of Lausanne and Geneva, Epalinges, Switzerland \\ ${ }^{2}$ Institute of Energy and Environmental Technology, Air Quality and Sustainable Nanotechnology Unit, \\ Duisburg, Germany \\ ${ }^{3}$ Centre for Human Exposure Science, Institute of Occupational Medicine, Edinburgh, United Kingdom \\ ${ }^{4}$ TNO, Rijswijk, The Netherlands \\ ${ }^{5}$ IOM Singapore, Chevron House, Singapore
}

\begin{abstract}
Different types of aerosolization and deagglomeration testing systems exist for studying the properties of nanomaterial powders and their aerosols. However, results are dependent on the specific methods used. In order to have well-characterized aerosols, we require a better understanding of how system parameters and testing conditions influence the properties of the aerosols generated. In the present study, four experimental setups delivering different aerosolization energies were used to test the resultant aerosols of two distinct nanomaterials (hydrophobic and hydrophilic $\mathrm{TiO}_{2}$ ). The reproducibility of results within each system was good. However, the number concentrations and size distributions of the aerosols created varied across the four systems; for number concentrations, e.g., from $10^{3}$ to $10^{6} \# / \mathrm{cm}^{3}$. Moreover, distinct differences were also observed between the two materials with different surface coatings. The article discusses how system characteristics and other pertinent conditions modify the test results. We propose using air velocity as a suitable proxy for estimating energy input levels in aerosolization systems. The information derived from this work will be especially useful for establishing standard operating procedures for testing nanopowders, as well as for estimating their release rates under different energy input conditions, which is relevant for occupational exposure.
\end{abstract}

Received 21 August 2015; accepted 16 October 2015.

(c) Yaobo Ding, Burkhard Stahlmecke, Araceli Sanchez Jimenez, Ilse L. Tuinman, Heinz Kaminski, Thomas A. J. Kuhlbusch, Martie van Tongeren, and Michael Riediker

This is an Open Access article. Non-commercial re-use, distribution, and reproduction in any medium, provided the original work is properly attributed, cited, and is not altered, transformed, or built upon in any way, is permitted. The moral rights of the named authors have been asserted.

Address correspondence to Michael Riediker, Institute for Work and Health (IST), Universities of Lausanne and Geneva, Route de la Corniche 2, Epalinges CH-1066, Switzerland. E-mail: Michael. Riediker@alumni.ethz.ch

\section{INTRODUCTION}

Engineered nanomaterials in powder form are widely used in modern technologies, such as in paint additives (Schaefer and Miszczyk 2013), catalysts (Svintsitskiy et al. 2013), nanocomposites (Gavrila-Florescu et al. 2012), functional ceramics (Zalite et al. 2008), and superconducting materials (Bansal et al. 2015). Particles accidentally aerosolized during production, handling, and storage of nanopowders in occupational settings may pose exposure risks to workers (Maynard et al. 2004; Kuhlbusch and Fissan 2006; Tsai et al. 2009; Wang et al. 2012). Nanoparticles have been shown to cause adverse health effects in human bodies via inhalation and subsequent translocation to secondary organs (Oberdoerster et al. 2004; Geiser and Kreyling 2010). Therefore, risks associated with exposure to engineered nanomaterials must be managed. A better understanding of how nanopowders behave during aerosolization is needed in order to establish proper safety control strategies in workplaces.

Dustiness, defined as the tendency of a powder material to generate airborne particles under an external energy input, has been tested by different systems to simulate powder handling processes in occupational settings. These experiments, which characterize airborne particle concentrations and size distributions, facilitate possible scenario predictions in exposure assessments. European Standard 15051, for measuring the dustiness of bulk materials, describes two reference testing procedures: the rotating drum method (EN 15051, part 2) and the continuous drop method (EN 15051, part 3) (Verlag 2013). However, these systems required large amounts of test materials that are not suitable for nanomaterials due to their costs and potential risks. A downscaled, modified test system has been developed combining continuous drop and a significantly smaller rotating drum, and this permits the use of smaller quantities of test materials (Schneider and Jensen 2008). Systems based on a vortex shaker, also offering the possibility of 
testing smaller quantities, have recently been studied in view of their application for dustiness characterization (Morgeneyer et al. 2013). Furthermore, Boundy et al. (2006) established an air jet dispersion method for testing the dustiness of pharmaceutical powders. The basic principle involves injecting powder through an orifice into a glass jar for subsequent characterization. It is noteworthy that these dustiness testing methods are different from deagglomeration tests, in that quantitative measurements of particle release are given (particle numbers or mass per unit nanopowder). Deagglomeration tests investigate the stability of nanoparticle agglomerates using different types of aerosolization and post-treatments on aerosolized particles subject to a range of energy levels. These tests provide qualitative results, but not quantitative ones, on how different forces trigger deagglomeration. Critical orifices have been used in these processes as means of applying high levels of shear forces (Stahlmecke et al. 2009; Sosnowski et al. 2014; Ding and Riediker 2015).

Whether tests based on different aerosolization processes deliver comparable results is, however, unknown. Indeed, measurements are influenced by the diverse parameters used in each aerosolization system. These include the system's intrinsic properties (e.g., associated energy levels; how that energy is applied, via shear force, impaction aerosolization, or dilution flow rates; compartment dimensions), environmental conditions (e.g., relative humidity), material characteristics (e.g., quantities tested and dustiness), and types of sampling device. Stronger deagglomeration processes may create airborne particles with a smaller mean size whereas less vigorous treatments may release larger particle agglomerates. Higher flow rates can dilute aerosols into lower concentrations. Raw material moisture content affects cohesive forces binding primary particles in powder agglomerates, and the dust generation rate is inversely proportional to this factor (Plinke et al. 1995). Furthermore, measurement devices that may themselves encourage deagglomeration during measurements (such as the Electrical Low Pressure Impactor) should be used with caution. Table $\mathrm{S} 1$ (see the online supplementary information [SI]) lists examples of the $\mathrm{TiO}_{2}$ nanopowder aerosol properties measured using different systems. Aerosol concentrations ranged from $100 \mathrm{\#} / \mathrm{cm}^{3}$ using a standard rotating drum method, to $10^{6} \mathrm{\#} / \mathrm{cm}^{3}$ using the vortex shaker method. The mode size of generated aerosols also differed from several hundred nanometers to a few microns.

The characterization of airborne nanoparticles generated from powders in occupational exposure assessment should, therefore, take into account the specific testing procedures. How different process characteristics influence measurements must be better understood. In the above aerosolization and deagglomeration methods, there was no common means of estimating associated energy levels. It is difficult to directly compare real-life exposure scenarios with the testing methods established to date, just as it is to predict aerosol properties resulting from a specific process and the subsequent exposure mechanism. A common method for comparing energy ranges across different systems is needed.

In the present study, four aerosolization and deagglomeration systems were used to test hydrophobic and hydrophilic $\mathrm{TiO}_{2}$ nanopowders. These systems provide relatively low (compared to treatments using critical orifices) but easily distinguishable energy input levels. We explored how system characteristics and test conditions modified aerosol characteristics such as concentration and size distribution. We also assessed if air velocity may be useful for estimating energy inputs in aerosolization systems. For this, a basic comparison of the systems presented was needed to facilitate ranking them for deagglomeration based on their methods. While some of the methods allow testing the stability of airborne agglomerates, in this article we only assessed the deagglomeration occurring during the aerosolization of the powder particles.

\section{MATERIALS AND TEST SETUPS}

\subsection{Materials}

Two nanomaterials in powder form were tested: hydrophobic titanium dioxide (NM103) and hydrophilic titanium dioxide (NM104) from the repository at the European Commission Joint Research Center (JRC-IHCP in Ispra). They had been stored in vials of $500 \mathrm{mg}$ or $100 \mathrm{~g}$ (depending on the test setup) in an inert atmosphere. Table 1 summarizes the main material characteristics (Rasmussen et al. 2014). The selection of these two materials as test powders was based on the rationale that the different surface coatings allow studying their effects on powder aerosolization and deagglomeration processes. The profiles of generated aerosols (size and concentration) are expected to differ due to distinct agglomeration levels caused by varied surface properties. Moreover, titanium dioxides are widely used in industrial sectors and have raised a high concern for human hazard risks (Shi et al. 2013).

TABLE 1

Physical and chemical properties of the tested materials

\begin{tabular}{lll}
\hline Name & $\begin{array}{c}\text { Titanium dioxide } \\
(\mathrm{NM103})\end{array}$ & $\begin{array}{c}\text { Titanium dioxide } \\
(\mathrm{NM} 104)\end{array}$ \\
\hline Composition & $\begin{array}{c}89 \% \mathrm{TiO}_{2}, \\
6.2 \% \mathrm{Al}_{2} \mathrm{O}_{3}\end{array}$ & $\begin{array}{c}89.8 \% \mathrm{TiO}_{2}, \\
6.2 \% \mathrm{Al}_{2} \mathrm{O}_{3}\end{array}$ \\
$\begin{array}{l}\text { Primary particle } \\
\text { size (XRD), } \mathrm{nm}\end{array}$ & 20 & 20 \\
$\begin{array}{l}\text { Surface modification } \\
\text { Hydrophobic }\end{array}$ & $\begin{array}{c}\text { Hydrophilic } \\
\text { (PHI) }\end{array}$ \\
$\begin{array}{l}\text { Specific area, } \mathrm{m}^{2} / \mathrm{g} \\
\text { Crystal structure }\end{array}$ & $\begin{array}{l}60 \\
\text { Rutile }\end{array}$ & 60 \\
Moisture content & $1.61 \%$ & Rutile \\
\hline
\end{tabular}

*Information from the manufacturer. 


\subsection{Test Setups}

Four different systems were used, featuring different types of aerosolization processes using a variety of energy inputs, and allowing a comparison of aerosol characteristics (particle number and size distribution) under different experimental conditions. Each system was developed or installed by one of the four partners and tested for the comparison study, using the above-described materials. Figure 1 shows schematic diagrams of the four measurement setups.

\subsubsection{System I-Fluidization Funnel}

A system based on fluidization was developed for continuous aerosolization of dry powders in small quantities (Figure 1a; Ding and Riediker 2015). Aerosolization is achieved inside a pressureresistant glass funnel. Filtered dry air is blown in from the bottom opening, activating the powder body. The aerosol created is diluted by another flow in a mixing chamber. Conditioned air with a different relative humidity can be introduced at this point to study its influence. A relative humidity $(\mathrm{RH})$ range from $2 \%$ to $90 \%$ can be controlled. Subsequently, the aerosol is transported into a large drum $(12 \mathrm{~L})$ from which online measurements and sample collection take place. A critical orifice can be installed in the chamber to study the stability of aerosol agglomerates but was not used in this study. Details of the sampling equipment are given in Table S2 (SI). To avoid particle losses, anti-static conductive tubes are used for particle transport between compartments. The measurement chamber is electrically grounded.

The system was initially flushed with filtered dry air to create a clean background $\left(<10 \mathrm{\#} / \mathrm{cm}^{3}\right)$. Powder quantities for each test were $250 \pm 10 \mathrm{mg}$. The aerosolization flow was set at $0.3-0.5 \mathrm{~L} / \mathrm{min}$ to maintain constant particle generation. The critical orifice was not installed for this study, and aerosol particles passed through a normal tube outlet into the measurement chamber. Particle concentration usually became stable after $20 \mathrm{~min}$ of aerosolization. Measurements continued for at least $30 \mathrm{~min}$ after this period. Relative humidity (RH) inside the system was $2 \pm 0.2 \%$, and the temperature was $20^{\circ} \mathrm{C}$. Two replicate tests were performed for each type of material.

\subsubsection{System II-Magnetic Stirrer}

A test rig using a magnetic stirrer in a pressurized beaker to activate powder materials is shown in Figure $1 \mathrm{~b}$ (Stahlmecke et al. 2009). A dry powder is aerosolized under constant carrier flow, and the aerosol generated is introduced into a mixing chamber for conditioning their RH (up to $90 \% \mathrm{RH}$ possible, validated up to $70 \% \mathrm{RH})$. After conditioning, the aerosol passes a)

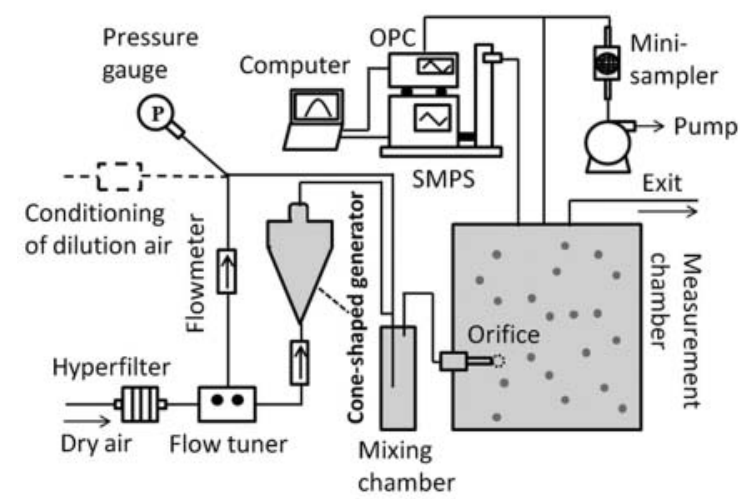

c)

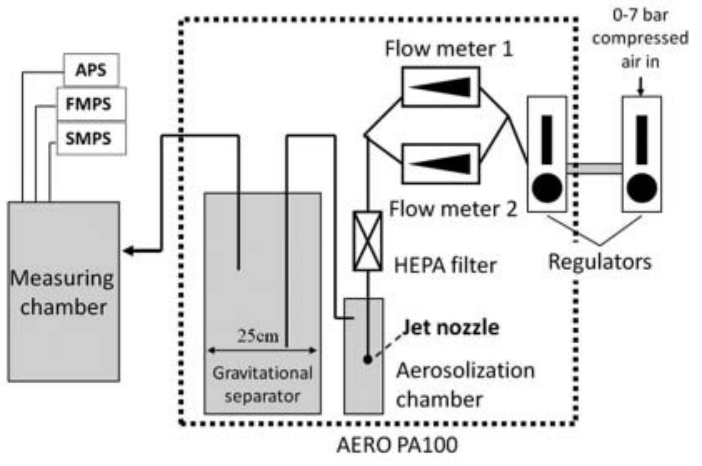

b)

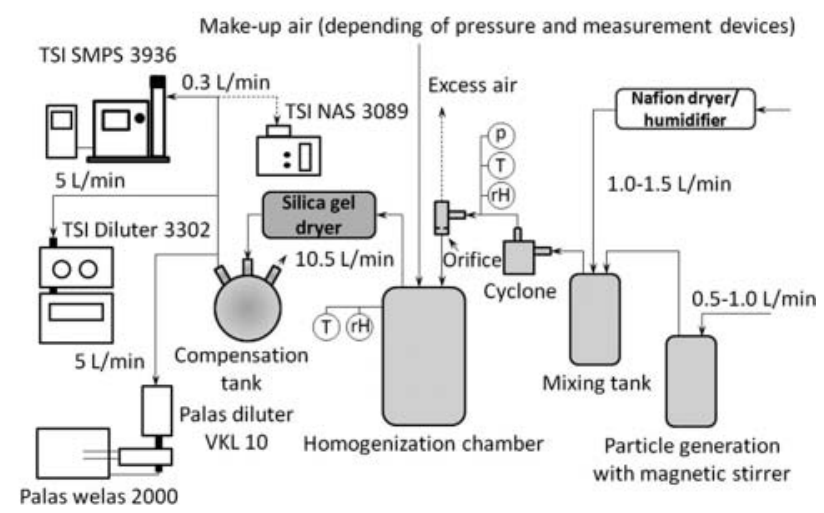

d)

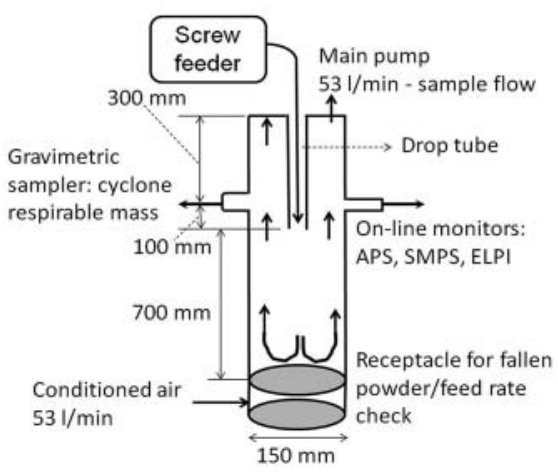

FIG. 1. Aerosolization systems tested: (a) fluidization funnel; (b) magnetic stirrer; (c) air jet aerosolizer; (d) continuous drop. 
a pre-separator (cyclone) to remove agglomerates above approximately $1.5 \mu \mathrm{m}$ (cut-off diameter). Finally, the aerosol is introduced into a homogenization chamber (volume $10 \mathrm{~L}$ ) either via normal tubing (zero overpressure) or via an orifice under various differential pressure conditions. The critical orifice was not installed in this study, which simulated the basic case of aerosolization for this setup. Anti-static conductive materials and tubes are used in the system to minimize particle losses. Airborne particles are characterized using online instrumentation sampling from the homogenization chamber (see Table S2, SI). Furthermore, they could be sampled on suitable substrates by using an electrostatic precipitator for subsequent scanning electron microscopic (SEM) analysis.

The powder volume tested in each experiment was $20 \mathrm{~cm}^{3}$. The background particle concentration inside the test system was recorded with a few measurement scans before the experiment. The magnetic stirrer's rotation speed (1000-1250 rpm, stirrer length: $30 \mathrm{~mm})$ inside the beaker $(250 \mathrm{~mL}$ high pressure glass bottle, Schott Duran glass bottle after DIN EN 1595) was controlled to produce constant powder agitation. Depending on the powder used, steel balls were occasionally used to assist the aerosolization process. The volume flow into the beaker was $0.5-1.0 \mathrm{~L} / \mathrm{min}$. The total flow volume needed by the measurement devices was about $10 \mathrm{~L} / \mathrm{min}$, thus an additional air flow was passed into the homogenization chamber to provide sufficient sampling flow. Sensors for temperature, humidity, and pressure were used to monitor experimental conditions. $\mathrm{RH}$ was below $2 \%$ in the tests. Measurements took approximately 30-45 min depending on the powder type. Two replicate tests were conducted for each type of material.

\subsubsection{System III-Air Jet}

The third system consisted of a commercial aerosolizer (Aero PA100, Model NA002, Particle Measuring Systems, United States) (Figure 1c). It generates aerosols from powder materials by applying high velocity air jets to the powder surface. A pressurized source of clean, dry air is connected and regulated at the input. The flow rate is controlled and monitored by two parallel flow meters. After passing through a high-efficiency particulare arrestance (HEPA) filter, the air is driven through a nozzle with three small holes to create high velocity jets for aerosolization. Aerosol generation strongly depends on the flow rate and how close the jet nozzle is to the powder surface. The aerosol passes through a gravitational separator $(10 \mathrm{~L})$, where large particle agglomerates are separated from the aerosol as a function of particle diameter and density. The aerosol is finally characterized by sampling from a measuring chamber. Details of characterization equipment are given in Table S2 (SI).

In this experiment, the aerosolization process used a $5 \mathrm{~cm}$ nozzle-to-powder distance. The aerosolizer was set at a constant $5 \mathrm{~L} / \mathrm{min}$ flow rate and was applied for $700 \mathrm{~s}$. Note that although the airflow was continuous, aerosol concentrations transported into the measuring chamber were not. Aerosol concentrations first increased, reached a maximum level, and subsequently dropped back to zero as the powder was consumed. Each experiment used $500 \mathrm{mg}$ of powder. The measuring chamber was ventilated after each run until the particle concentration was below $10 \# / \mathrm{cm}^{3}$ as measured using a condensation particle counter (CPC). During all runs, $\mathrm{RH}$ in the measuring chamber was $26 \pm 2 \%$ and the temperature was 17 $\pm 1^{\circ} \mathrm{C}$. Two replicate tests were carried out for each type of material.

\subsubsection{System IV-Continuous Drop}

The continuous drop method, often used as a reference tool for testing the dustiness of dry powders, was also used in this study (CEN 2013). The aerosolization process is shown in Figure $1 \mathrm{~d}$. The powder to be tested is placed in a screw feeder that drops the powder into the drop chamber at a constant feed rate. The dropping powder meets an upward air flow of $53 \mathrm{~L} / \mathrm{min}$ $(0.05 \mathrm{~m} / \mathrm{s})$ introduced from the cylinder chamber floor, creating turbulence in the particle surroundings. The aerosol generated is sampled above the drop tube. Relative humidity and temperature are adjusted by introducing conditioned air into the system.

The feed rate $(2.8 \mathrm{~g} / \mathrm{min})$ was adjusted and measured before each experiment. The cyclone and drop cylinder were flushed by running clean air through them at $20^{\circ} \mathrm{C}$ and at $50 \% \mathrm{RH}$ for $10 \mathrm{~min}$. Next, the feeder was run continuously for $1 \mathrm{~min}$, before measurements started which lasted $5 \mathrm{~min}$. Details of characterization equipment are given in Table S2 (SI). Two replicate tests were carried out for each type of material.

\subsection{Characterization Methods}

Scanning mobility particle sizer (SMPS), optical particle counter (OPC), and aerodynamic particle sizer (APS) were used to measure particle number concentration and size distribution. The instruments used in the different setups are summarized in Table S2 (SI). An inter-comparison of the SMPS settings used in the test methods is given by Table S3 (SI). It is noteworthy that the equivalent diameters determined by these different devices are not the same, due to different measurement techniques used. This should be considered when constructing and interpreting the size distributions. The SMPS determines the electrical mobility of airborne particles. The OPC characterizes particle diameter by their optical properties and the light scattering principle. The APS classifies the particles according to the aerodynamic diameter.

\subsection{Estimation of Energy Input}

Although the four measurement systems shared certain similarities, they differed in several aspects, shown in Table 2. For 
TABLE 2

General comparison of test setups

\begin{tabular}{llllll}
\hline System & Material quantity & $\begin{array}{c}\text { Flow rate, } \\
\text { L/min }\end{array}$ & Relative humidity & $\begin{array}{c}\text { Aerosolization } \\
\text { mechanism }\end{array}$ & $\begin{array}{c}\text { Relative velocity (energy level), } \\
\mathrm{m} / \mathrm{s}\end{array}$ \\
\hline Funnel & $250 \pm 10 \mathrm{mg}$ & $1.5-2$ & $2 \pm 0.2 \%$ & Blowing & $1.32-2.20$ (medium) \\
Stirrer & $20 \mathrm{~cm}^{3}(5-8 \mathrm{~g})$ & 2 & $<2 \%$ & Mechanical stirring & $0-1.96$ (low-medium) \\
Air jet & $500 \pm 10 \mathrm{mg}$ & 5 & $26 \pm 2 \%$ & Blowing & 14 (high) \\
Drop & $2.8 \mathrm{~g} / \mathrm{min}$ & 53 & $50 \%$ & Air friction & $0.003-0.88$ (low) \\
\hline
\end{tabular}

example, aerosolization energies differed significantly between systems. As an indirect parameter of energy, the relative velocity between the aerosolization air flow and the powder particles was used to compare the different systems' energy levels. The funnel setup used $0.3-0.5 \mathrm{~L} / \mathrm{min}$ air flow to aerosolize the powder, creating an air velocity of $1.32-2.20 \mathrm{~m} / \mathrm{s}$ at the funnel's bottom hole (2.5 $\mathrm{mm}$ in diameter). The rotating magnetic stirrer (a solid stick $3 \mathrm{~cm}$ long) in the pressurized beaker had a maximum linear velocity of $1.57-1.96 \mathrm{~m} / \mathrm{s}$ at both ends (assumed to be the aerosolization air flow speed), as calculated based on a speed of 1000-1250 rpm. In the air jet system, the powder was kept $5 \mathrm{~cm}$ from the air nozzle delivering a $5 \mathrm{~L} / \mathrm{min}$ flow rate. The air speed measured at $5 \mathrm{~cm}$ away from the nozzle (simulating the scenario when the flow reaches the powder surface) was $14 \mathrm{~m} / \mathrm{s}$. In the continuous drop method, particle settling velocity was calculated as the relative speed to air. For particles with a diameter of $10 \mu \mathrm{m}$, the Stokes's law applies for determining particle settling velocities (Hinds 1982). For particles with a diameter of $100 \mu \mathrm{m}$, with a Reynolds number larger than 1.0, a modified equation is used to calculate the settling velocity (Hinds 1982). Settling velocities were $0.003 \mathrm{~m} / \mathrm{s}$ and $0.88 \mathrm{~m} / \mathrm{s}$ for $10 \mu \mathrm{m}$ and $100 \mu \mathrm{m}$ particles, respectively. Particles smaller than $10 \mu \mathrm{m}$ have even slower settling speeds.

Based on these calculations, Table 2 provides a rough (low, medium, or high) ranking of the energy input levels in the aerosolization methods used. Other system characteristics that could potentially alter the properties of generated aerosols are also listed.

\subsection{Data Analysis}

Number concentrations in the size range below $1 \mu \mathrm{m}$, as well as the mode diameters in the aerosols generated, were compared across the different systems. Broader size distribution spectrums were plotted by combining SMPS and OPC (or APS) data (effective density used: air jet and drop systems, $1 \mathrm{~g} / \mathrm{cm}^{3}$. refractive index used: Funnel, 1.59; Stirrer, 2.56). The data units from the optical particle counters were converted into $d N / d \log D p\left[\# / \mathrm{cm}^{3}\right]$, in order to compare size channels with the different widths used in the other devices. Particle size distributions from replicate tests were compared to estimate each system's robustness. Spectrums were normalized to the total particle number in the size range considered (dependent on the specific system). This allowed a better comparison of the size distributions in different concentrations. The mode size(s) of aerosols is also plotted against the velocity of the aerosolization flows in the different systems, facilitating the analysis of this parameter's potential influence on aerosol properties. The size distributions of aerosols with different surface coatings are also plotted.

\section{RESULTS AND DISCUSSION}

\subsection{Particle Number Concentration and Mode Diameter}

A comparison of number concentrations in the aerosols generated from the different systems is shown in Figure 2 (left). The SMPS and APS/OPC data were used, and total

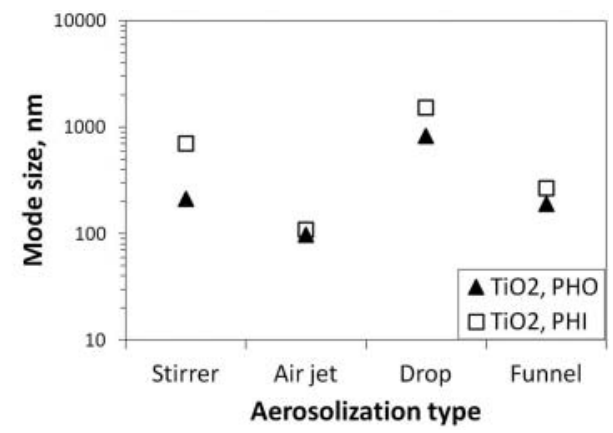

FIG. 2. Comparison of total particle number (34-965 nm) (left) and mode diameter (right). 
particle numbers were compared for a common same size covered by all the systems. Particle numbers varied across a large range, from about $200 \# / \mathrm{cm}^{3}$ to $100,000 \# / \mathrm{cm}^{3}$. The funnel and drop setups produced lower concentrations in the aerosols of both materials compared to the other two methods. Hydrophobic powder aerosols showed higher particle concentrations than hydrophilic powder aerosols in three methods, but not with the air jet system-it produced higher numbers of hydrophilic particles. In all four systems, the differences in particle numbers for the different materials were about one order of magnitude.

The mode diameter of the aerosols generated is also shown in Figure 2 (right). The results varied greatly: from about $100 \mathrm{~nm}$ to above $1 \mu \mathrm{m}$. The largest mode diameters resulted from the continuous drop method; the smallest mode diameter came from the air jet setup. The hydrophilic particles usually had a larger mode size than the hydrophobic particles, however the diameters for the two materials were similar in the air jet system. It is noteworthy that a second mode was observed in the size distribution of the hydrophobic aerosol generated in the drop method. This is discussed in the following sections.

Particle number concentrations in experimentally-generated aerosols have a close relationship to the system characteristics and testing conditions. The amount of raw materials used indicates how many particles are available to be aerosolized. Energy input may affect the level of deagglomeration of the powder particles. High energy processes are more likely to thoroughly break-up the powder agglomerates, thus generating aerosols with a high particle number concentration. Furthermore, the volume flow rate also modifies particle number. For the same amount of available particles, a higher flow rate dilutes the aerosol into a lower number per unit volume. In order to study deagglomeration processes, it would be key to maintain the airborne particle concentration within a range that is above the detection limit of the measurement instruments, but also not sufficiently high as to promote immediate secondary effects (e.g., re-agglomeration in the airborne state). The systems used in this study worked in suitable concentration ranges, which allowed a comparison of the deagglomeration effects in the different setups.

The system parameters and testing conditions varied between the experiments. The associated energy levels were highest for the air jet system, as calculated in the method section, and this might be responsible for the high particle generation of the hydrophilic powder, in spite of low material consumption $(500 \mathrm{mg}$ ). On the other hand, for the drop method, although the quantity of material used was high $(\sim 2.8 \mathrm{~g})$, the particle number was low due to the relatively small energy input for aerosolization. In comparison, the stirrer system seemed to somehow balance these factors. The funnel setup, with low material use $(250 \mathrm{mg})$ and a moderate aerosolization energy, worked in low concentration ranges under the given aerosolization flows. Similarly, the vortex shaker method has generated concentrations in $300-2000 \# / \mathrm{cm}^{3}$ range using only $0.25-1 \mathrm{~cm}^{3} \mathrm{TiO}_{2}$ powders (Ogura et al. 2009). The air flow rate was 5-20 times higher in the drop system than in the other systems. This may have contributed to the low number concentration observed. In general, the high particle concentrations obtained in our experiments were comparable to those for ultrafine $\mathrm{TiO}_{2}$ in the combined single drop-rotating drum method (up to $10^{6} \# / \mathrm{cm}^{3}$ ) (Schneider and Jensen 2008).

Aerosolization time is another factor influencing particle number concentration. This is especially true for systems showing decreasing particle generation over time (Dahmann and Monz 2011). In the air jet experiments, particle concentrations reached maximum values after a few minutes of aerosolization. Particle numbers then gradually decreased to background levels. Similar patterns have been observed: brief initial bursts, decaying rates during rotation, and then constant rates (Schneider and Jensen 2008). The difference in the air jet method was that material quantities used were small, thus powder was rapidly consumed, and then concentrations dropped to a very low level. In comparison, the funnel system was shown to be able to maintain stable concentrations over longer time periods (>30 min) (Ding and Riediker 2015). This was also the case for the stirrer system in the present work.
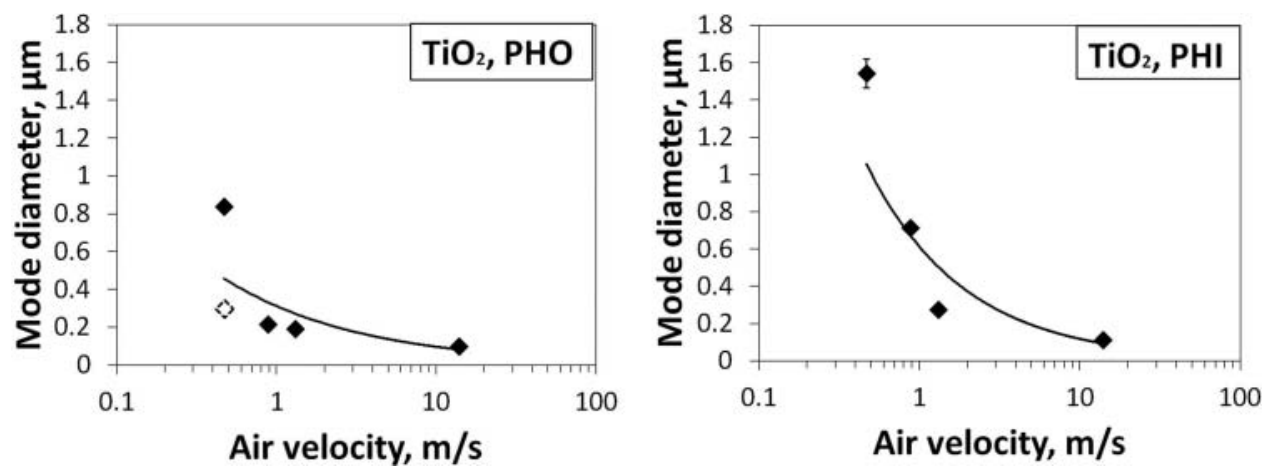

FIG. 3. Influence of aerosolization flow velocity on mode diameter of generated aerosols (fitted curves are added to show the general patterns; white dot on left graph represents secondary mode diameter for the drop method). 


\subsection{Effect of Air Velocity on Aerosol Diameter}

The energy inputs during different aerosolization processes were ranked based on the relative velocity of activation air flow as described above. A comparison of the air velocityaerosol size relationship is given in Figure 3. The both mode sizes measured by the SMPS in the drop system are included. Particle diameters were shown to be inversely proportional to the velocity of aerosolization flows. Higher air speeds generated smaller mode sizes. Particle sizes decreased rapidly in the velocity range up to $1 \mathrm{~m} / \mathrm{s}$, but size reduction slowed down at higher speeds. Similar patterns were seen for both materials. The hydrophilic particles experienced larger decreases in mode size as air speed increased than did the hydrophobic particles.

The mode diameter of aerosol particles was earlier reported to be associated with the energy level during powder aerosolization: the size of airborne particle agglomerates was smaller under higher shear forces (Stahlmecke et al. 2009; Ding and Riediker 2015). This was explained by drag from the air current, which acted as a major deagglomerating force in these processes. The drag force is proportional to the velocity and diameter of the particle (Hinds 1982):

$$
F_{D}=\frac{3 \pi \eta v D}{C}
$$

where $\eta$ is the air viscosity; $v$ is the particle velocity relative to air; $D$ is the dynamic shape factor; and $C$ is the slip correction factor. The shape factor is constant for a given particle, and it was set equal to 1 for simplicity matters. The Cunningham slip effect becomes significant when particle size is below $10 \mu \mathrm{m}$. Drag forces differ for particle agglomerates with different diameters. The values calculated against particle size at the average velocities in the systems tested are shown in Figure 4. For $1 \mu \mathrm{m}$ particles, forces ranged from 0.06 to $2.05 \mathrm{nN}$.

The dominant mechanism by which uncharged particles form agglomerates when stored as powders is direct contact (Turki and Fatah 2008), bonding individual particles by van der Waals (VDW) force. This interparticular force can be

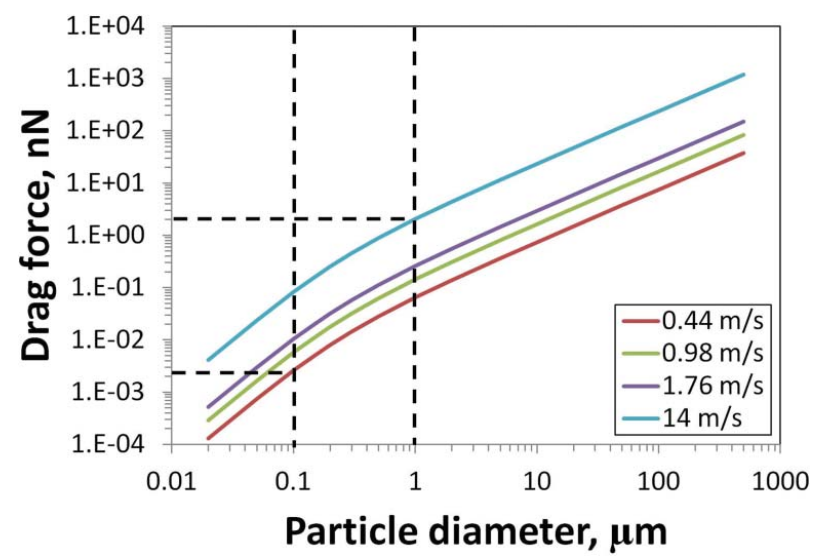

FIG. 4. Drag force-particle size relationship for the various relative velocities in the tests.

calculated as below (Hamaker 1937)

$$
F=-\frac{A}{D_{1}} F_{y}(x), y=\frac{D_{2}}{D_{1}}, x=\frac{r}{D_{1}}
$$

where $A$ is the Hamaker constant; $D_{1}$ is the diameter of the smaller particle; $D_{2}$ is the diameter of the larger particle; and $r$ is the distance between the two particles.

For two spherical particles of the same diameter $(y=1)$, when $x<<1$, then approximately

$$
F_{1}(x)=-\frac{1}{24} \frac{1}{x^{2}}
$$

The contact distances of two primary particles can be viewed as the material's VDW radius. At this distance, separation between the particles reaches an equilibrium where the interfacial potential is minimal (Cheng et al. 2002). For titanium, the value is $0.215 \mathrm{~nm}$ (Batsanov 2001). The diameter of primary particles in our tests was $20 \mathrm{~nm}\left(D_{1}=D_{2}\right)$. The Hamaker constant $A$ for $\mathrm{TiO}_{2}-\mathrm{TiO}_{2}$ (rutile) interaction in the air is $15.3 \times 10^{-20} \mathrm{~J}$ (Bergström 1997). The result of VDW force interaction was $F_{\mathrm{VDW}}=2.64 \mathrm{nN}$ for $r=0.215 \mathrm{~nm}, D=$ $20 \mathrm{~nm}$.
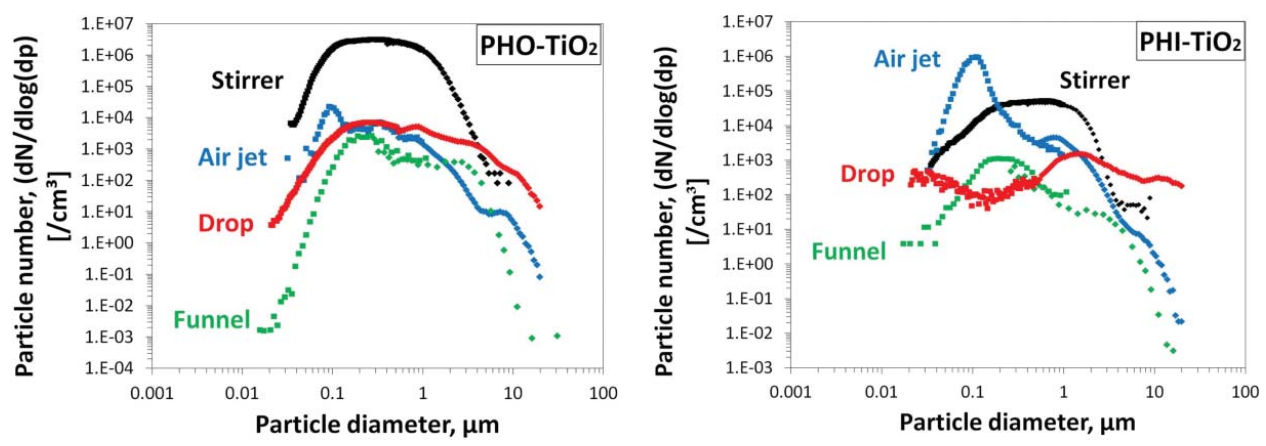

FIG. 5. Comparison of full particle number size distributions for the different systems. 
In comparison, the drag forces created in our different test setups were in the range of $2 \times 10^{-3} \sim 2 \mathrm{nN}$ for particle diameters of $0.1-1 \mu \mathrm{m}$ (Figure 4). Although the drag comprises small forces, up to three orders of magnitude lower than the calculated VDW force, it can still affect the deagglomeration process. In a study using numerical simulation to investigate the dispersion of $\mathrm{TiO}_{2}$ nanoparticle aggregates under shear flow, it was found that the aggregates started to deagglomerate when the ratio of fluid force to the interparticle force was over 0.001 (Nishiyama et al. 2013). Thus, the shear force ingredients created in our experiments may be responsible for the different mode diameters of the aerosols generated. Higher air velocities induce stronger drag on the particle agglomerates, which leads to higher deagglomeration efficiencies by overcoming interparticular binding forces. As a follow-up, we have carried out further work on quantifying the effects of air speed by incorporating critical orifices in two of the systems, which expands on the present study into a consideration of a much broader energy range.

\subsection{Particle Size Distribution}

Particle number size distributions from the aerosols of different systems, including SMPS and OPC (or APS) data, are compared in Figure 5. The peaks in the size distributions were located in different size ranges. However, the deviations seemed to be smaller for the hydrophobic aerosols than for their hydrophilic counterparts. Comparing the two powders by system, higher peaks were generally shown for the hydrophobic particles than for the hydrophilic particles, except for the air jet system. The peaks created using the air jet and the funnel methods were sharper; they were relatively broader for the other two systems. Two particle size modes were observed for the hydrophobic aerosol in the continuous drop system (Figure 5, left): one above $1 \mu \mathrm{m}$ and another around $300 \mathrm{~nm}$ (measured by SMPS). Small variations were noted when combining data points obtained using different measurement equipments. However, this was only to be expected because of their different operating principles.

The varied shapes of the size distribution spectrums can be attributed to the different deagglomeration levels in the test setups. At low energy input, powder particles were partially deagglomerated, generating aerosols with a large mode size. Using large amounts of materials may alter local interactions between the air flow and the powder particles, which may lead to the reduced dispersibility of the powder agglomerates. How agglomerate size in powder fluidizations depends on parameters including gas velocity and energy input has been described previously (Zhu et al. 2005; van Ommen et al. 2012). In contrast, higher drag forces created in the air jet system broke agglomerates down to the sizes for which associated drags become comparable to the interparticle binding force (as discussed in previous sections). Furthermore, the funnel setup-which resembles a fluidized bed-features another deagglomeration mechanism: collisions between flowing particles (Turki and Fatah 2008; van Ommen et al. 2010). These conditions promoted sharper peaks in the size distribution of the aerosols generated.

The two modes in the particle size spectrum observed in the drop system may be due to the low energy input. Indeed, a particle size distribution in powders is usually bimodal (Andrès et al. 1996; HORIBA 2014). Primary particles form submicron and micro-sized agglomerates. Small particles are readily aerosolized, but big particles need more energy to be deagglomerated. The mode size measured in the $200-300 \mathrm{~nm}$ range may be directly due to small, easily aerosolized particles, whereas the mode in the 1-2 $\mu \mathrm{m}$ range was the result of particles broken down from larger powder agglomerates under low shear forces. Other investigators of $\mathrm{TiO}_{2}$ have observed similar bimodal behavior in submicron and micron ranges when using rotating drum methods, as shown in Table S1 (SI). Energy input into those systems was considered low, since the rotation speeds used were $4 \mathrm{rpm}$ (Tsai et al. 2009) and $11 \mathrm{rpm}$ (Schneider and Jensen 2008). This process shares some similarities with the drop method, as amounts of powder are raised to a certain height and fall back down. Moreover, Dahmann and Monz (2011) showed that nanopowders tested in their continuous drop experiments typically had bimodal distributions. In comparison, monodispersed size distributions have been more common in high energy processes, such as vortex shaker systems (Ogura et al. 2009; Morgeneyer et al. 2013).

\subsection{Reproducibility}

The results obtained in replicate tests from different systems are summarized in Table S4 (SI). Variations in absolute number concentrations were generally small, but some cases with several-fold differences were also observed. Overall the results were still within the same order of magnitude. Total particle number concentrations were calculated for the size range below $1 \mu \mathrm{m}$, with the exception of the drop method, which had larger mode sizes for its aerosolized particles. The mode diameters from replicate tests were very similar (SD < $8.3 \%$ ). Figure S1 (SI) compares the size distribution spectrums, at relative scales, for hydrophilic $\mathrm{TiO}_{2}$. The particle fractions in certain size ranges varied slightly. A relatively larger difference in peak heights was seen using the drop method. In general, the reproducibility of the aerosols generated by the four systems was good, facilitating a robust comparison of the different methods.

\subsection{Effects of Material Surface Coatings}

Relative particle number distributions were compared for the two materials with different surface coatings (Figure S2, SI). In general, the mode diameters were larger for the hydrophilic $\mathrm{TiO}_{2}$ than for hydrophobic. The differences between the 
two materials were more significant in the stirrer $(\mathrm{RH} \sim 2 \%)$ and the drop systems (RH $\sim 50 \%)$ than that in the air jet (RH $\sim 26 \%$ ) and the funnel systems (RH $\sim 2 \%$ ), even though the $\mathrm{RH}$ in the stirrer system was very low. This can possibly be explained by the shear forces during aerosolization. The differences were small for the two systems with the highest air velocities and significant for the two systems with the lowest velocities. In the drop system, a bi-modal size spectrum was observed for the hydrophobic $\mathrm{TiO}_{2}$, which exhibited a high particle fraction in 200-300 $\mathrm{nm}$ range.

Particles with hydrophilic surfaces absorb water more easily than particles with hydrophobic coatings. Both environmental humidity and the raw powder's moisture content can contribute to the formation of water menisci between individual particles. In mid-range RH (40\%-70\%), it has been shown that the pull-off force (maximum attractive force between particles) on a hydrophilic surface increased with the increasing humidity (Israelachvili 2011). On mica surfaces, this force was several times larger in the capillary regime than in the pure VDW regime. This might explain the differences in mode sizes and number concentrations between our two aerosols, both in the drop system with $50 \% \mathrm{RH}$, and in the dry stirrer and funnel systems. However, with higher energy inputs, the difference in particle size was smaller, as seen in the air jet system. The drag force level created using this method may overcome the additional capillary adhesion from the water layer, thus triggering deagglomeration. In this case, the effect of a hydrophilic surface is compromised by a sufficiently high shear force.

\section{CONCLUSIONS}

The present study tested $\mathrm{TiO}_{2}$ nanomaterial powders with different surface coatings (one hydrophilic and one hydrophobic) using four different aerosolization and deagglomeration systems. These generated stable aerosols for measurement, and the results obtained in each system showed good reproducibility. However, significant variations in aerosol properties, such as number concentration and size distribution, were observed in the different setups. The hydrophilic aerosol of $\mathrm{TiO}_{2}$ tended to have a larger particle size than its hydrophobic counterpart. However, processes associated with high energy input levels seem to reduce the influence of surface properties on particle size distributions. Finally, the particle size was shown to be inversely related to the velocity of the aerosolization air flows.

The test setups used very different air flow rates, raw material quantities, aerosolization mechanisms, and associated energy levels. The varied results from the same materials indicate that the characterization of nanoparticle release should take into account specific testing protocols. The data obtained from each of the different systems offer suggestions as to which scenarios they could model most appropriately and how standard operating procedures (SOPs) should be adapted with regard to specific tests. For example, the drop method could be used to determine a material's propensity to be aerosolized (dustiness) in low energy processes, thanks to its ability to measure the mass fraction of samples that become airborne. In contrast, the three other methods are more useful and valuable for the study of agglomerate stability in high energy processes. They are also useful for the determination of particle size distributions in powder characterizations.

The relative velocity of aerosolization air flow was used for a rough comparison of system energies to study if aerosol properties are affected by this parameter. More precise estimations of energy input level would take into account specific aerosolization method (the way external energies are applied). This aspect can be further explored in future experiments. In the present work, the significant influence of air velocity on aerosol particle diameter indicates that this parameter might be a good indicator for the energy levels associated with a variety of industrial processes. It is especially applicable to the handling of nanomaterial powders in occupational settings, where air velocities can be easily assessed from workers' operational activities and behaviors. For example, air speeds when handling powders in a laboratory can be estimated from fume hood flow patterns and other process parameters such as transfer distances, pouring heights, or mixing rates. The "microenvironment" surrounding powder particles during filling and packaging at manufacturing sites involves air current movements, assessed using local conditions (e.g., ventilation or wind speed) and workers' operational procedures. The values calculated can then be compared with those from laboratory testing methods, in order to predict aerosol properties that might result from such scenarios. This is especially useful when field measurement data are lacking, but the risk level is considered high and in need of assessment. As a further step, this metric could be used to rank the potential for nanoparticle release in different industrial activities and processes.

\section{FUNDING}

The research leading to these results has received funding from the European Research Council under the European Union's Seventh Framework Programme (FP/2007-2013)/ ERC Grant Agreement no. 263215.

\section{SUPPLEMENTAL MATERIAL}

Supplemental data for this article can be accessed on the publisher's website.

\section{REFERENCES}

Andrès, C., Réginault, P., Rochat, M. H., Chaillot, B., and Pourcelot, Y., (1996). Particle-Size Distribution of a Powder: Comparison of Three Analytical Techniques. Int. J. Pharmaceut., 144(2):141-146. 
Bansal, N. P., Goldsby, J. C., Rogers, R. B., Susner, M. A., and Sumption, M. D., (2015). Chemical Synthesis of Superconducting MgB2 Nanopowder. J. Alloy. Compound., 622(0):986-988.

Batsanov, S. S., (2001). Van der Waals Radii of Elements. Inorg. Mater., 37 (9):871-885.

Bergström, L., (1997). Hamaker Constants of Inorganic Materials. Adv. Colloid Interface Sci., 70(0):125-169.

Boundy, M., Leith, D., and Polton, T. (2006). Method to Evaluate the Dustiness of Pharmaceutical Powders. Annals of Occupational Hygiene, 50(5):453-458.

CEN. (2013). FprEN 15051 Workplace Exposure: Measurement of the Dustiness of Bulk Materials; Part 1: Requirements and Choice of Test Methods; Part 2: Rotating Drum Method; Part 3: Continuous Drop Method. European Committee for Standardization, Brussels, Belgium.

Cheng, W., Dunn, P. F., and Brach, R. M., (2002). Surface Roughness Effects Onmicroparticle Adhesion. J. Adhesion, 78(11):929-965.

Rasmussen, K., Mast, J., De Temmerman, P.-J., Verleysen, E., Waegeneers, N., Van Steen, F., Pizzolon, J. C., De Temmerman, L., Van Doren, E., Jensen, K. A., Birkedal, R., Levin, M., Nielsen, S. H., Koponen, I. K., Clausen, P. A., Kofoed-Sørensen, V., Kembouche, Y., Thieriet, N., Spalla, O., Giuo,t C., Rousset, D., Witschger, O., Bau, S., Bianchi, B., Motzkus, C., Shivachev, B., Dimowa, L., Nikolova, R., Nihtianova, D., Tarassov, M., Petrov, O., Bakardjieva, S., Gilliland, D., Pianella, F., Ceccone, G., Spampinato, V., Cotogno, G., Gibson, P., Gaillard, C., and Mech, A. (2014). Titanium Dioxide, NM-100, NM-101, NM-102, NM-103, NM-104, NM-105: Characterisation and Physico-Chemical Properties.

Dahmann, D., and Monz, C., (2011). Determination of Dustiness of Nanostructured Materials. Gefahrst. Reinhalt. L., 71(11/12):481-487.

Ding, Y., and Riediker, M., (2015). A System to Assess the Stability of Airborne Nanoparticle Agglomerates Under Aerodynamic Shear. J. Aerosol Sci., 88(0):98-108.

Gavrila-Florescu, L., Sandu, I., Stan, A., Dutu, E., and Voicu, I., (2012). Laser Synthesized Nanopowders for Polymer-Based Composites. Appl. Surf. Sci., 258(23):9260-9262.

Geiser, M., and Kreyling, W., (2010). Deposition and Biokinetics of Inhaled Nanoparticles. Part. Fibre Toxicol., 7(1):2.

Hamaker, H. C., (1937). The London-Van Der Waals Attraction Between Spherical Particles. Physica, 4(10):1058-1072.

Hinds, W., (1982). Aerosol Technology - Properties, Behaviour, and Measurement of Airborne Particles (2nd ed.). John Wiley \& Sons, New York.

HORIBA (2014). A Guidebook to Particle Size Analysis. HORIBA Instruments, INC, Irvine, CA, USA.

Israelachvili, J. N., (2011). Van der Waals Forces Between Particles and Surfaces, in Intermolecular and Surface Forces (3rd ed.), J. N. Israelachvili, ed., Academic Press, San Diego, pp. 253-289.

Kuhlbusch, T. A., and Fissan, H., (2006). Particle Characteristics in the Reactor and Pelletizing Areas of Carbon Black Production. J. Occup. Environ. Hyg., 3(10):558-567.

Maynard, A., Baron, P., Foley, M., Shvedova, A., Kisin, E., and Castranova, V., (2004). Exposure to Carbon Nanotube Material: Aerosol Release During the Handling of Unrefined Single Walled Carbon Nanotube Material. J. Toxic. Environ. Health, 67:87-107.

Morgeneyer, M.,, Le Bihan, O., Ustache, A., and Aguerre-Chariol, O., (2013). Experimental Study of the Aerosolization of Fine Alumina Particles From Bulk by a Vortex Shaker. Powder Technol., 246(0): 583-589.

Nishiyama, T., Inamuro, T., and Yasuda, S., (2013). Numerical Simulation of the Dispersion of Aggregated Brownian Particles Under Shear Flows. Comput. Fluids, 86(0):395-404.
Oberdoerster, G., Sharp, Z., Atudorei, V., Elder, A., Gelein, R., Kreyling, W., and Cox, C., (2004). Translocation of Inhaled Ultrafine Particles to the Brain. Inhal. Toxicol., 16:437-445.

Ogura, I., Sakurai, H., and Gamo, M. (2009). Dustiness Testing of Engineered Nanomaterials. J. Phys.: Conf. Ser., 170:012003.

Plinke, M. A. E., Leith, D., Boundy, M..G., and Löffler, F., (1995). Dust Generation from Handling Powders in Industry. Am. Ind. Hyg. Assoc. J., 56 (3):251-257.

Schaefer, K., and Miszczyk, A., (2013). Improvement of Electrochemical Action of Zinc-Rich Paints by Addition of Nanoparticulate Zinc. Corros. Sci., 66(0):380-391.

Schneider, T., and Jensen, K., (2008). Combined Single-Drop and Rotating Drum Dustiness Test of Fine to Nanosize Powders Using a Small Drum. Ann. Occup. Hyg., 52(1):23-34.

Shi, H., Magaye, R., Castranova, V., and Zhao, J., (2013). Titanium Dioxide Nanoparticles: A Review of Current Toxicological Data. Part. Fibre. Toxicol., 10:15.

Sosnowski, T. R., Giżyńska, K., and Żywczyk, Ł. (2014). Fluidization and Break-up of Powder Particle Aggregates During Constant and Pulsating Flow in Converging Nozzles. Colloid. Surf. A, 441(0): 905-911.

Stahlmecke, B., Wagener, S., Asbach, C., Kaminski, H., Fissan, H., and Kuhlbusch, T., (2009). Investigation of Airborne Nanopowder Agglomerate Stability in an Orifice Under Various Differential Pressure Conditions. $J$. Nanopart. Res., 11(7):1625-1635.

Svintsitskiy, D. A., Chupakhin, A. P., Slavinskaya, E. M., Stonkus, O. A., Stadnichenko, A. I., Koscheev, S. V., and Boronin, A. I., (2013). Study of Cupric Oxide Nanopowders as Efficient Catalysts for LowTemperature CO Oxidation. J. Mol. Catal. A: Chem., 368-369(0): 95-106.

Tsai, C., Wu, C., Leu, M., Chen, S., Huang, C., Tsai, P., and Ko, F., (2009). Dustiness Test of Nanopowders Using a Standard Rotating Drum with a Modified Sampling Train. J. Nanopart. Res., 11(1):121-131.

Tsai, S. J., Ada, E., Isaacs, J. A., and Ellenbecker, M. J., (2009). Airborne Nanoparticle Exposures Associated with the Manual Handling of Nanoalumina and Nanosilver in Fume Hoods. J. Nanopart. Res., 11(1): $147-161$.

Turki, D., and Fatah, N., (2008). Behavior and Fluidization of the Cohesive Powders: Agglomerates Sizes Approach. Braz. J. Chem. Eng., 25(04): 697-711.

van Ommen, J. R., King, D. M., Weimer, A., Pfeer, R., and van Wachem, B., (2010). Experiments and Modelling of Micro-Jet Assisted Fluidization of Nanoparticles, in Paper Presented at The 13th International Conference on Fluidization - New Paradigm in Fluidization Engineering. ECI Digital Archives, Engineering Conferences International, New York, NY, USA.

van Ommen, J. R., Valverde, J., and Pfeffer, R., (2012). Fluidization of Nanopowders: A Review. J. Nanopart. Res., 14(3):1-29.

Verlag, B., (2014). European Norm 15051, Workplace Exposure-Measurement of the Dustiness of Bulk Materials, British Standards Institution, London, UK

Wang, J., Asbach, C., Fissan, H., Hulser, T., Kaminski, H., Kuhlbusch, T. A. J., and Pui, D. Y. H., (2012). Emission Measurement and Safety Assessment for the Production Process of Silicon Nanoparticles in a Pilot-Scale Facility. J. Nanopart. Res., 14:759.

Zalite, I., Zilinska, N., and Kladler, G., (2008). SiAlON Ceramics From Nanopowders. J. Eur. Ceram. Soc., 28(5):901-905.

Zhu, C., Yu, Q., Dave, R. N., and Pfeffer, R., (2005). Gas Fluidization Characteristics of Nanoparticle Agglomerates. AIChE J., 51(2):426-439. 